\title{
Combinatorial Reciprocity for Monotone Triangles
}

\author{
Ilse Fischer and Lukas Riegler $\|^{\dagger}$
}

Fakultät für Mathematik, Universität Wien, Austria

\begin{abstract}
The number of Monotone Triangles with bottom row $k_{1}<k_{2}<\cdots<k_{n}$ is given by a polynomial $\alpha\left(n ; k_{1}, \ldots, k_{n}\right)$ in $n$ variables. The evaluation of this polynomial at weakly decreasing sequences $k_{1} \geq k_{2} \geq \cdots \geq$ $k_{n}$ turns out to be interpretable as signed enumeration of new combinatorial objects called Decreasing Monotone Triangles. There exist surprising connections between the two classes of objects - in particular it is shown that $\alpha(n ; 1,2, \ldots, n)=\alpha(2 n ; n, n, n-1, n-1, \ldots, 1,1)$. In perfect analogy to the correspondence between Monotone Triangles and Alternating Sign Matrices, the set of Decreasing Monotone Triangles with bottom row $(n, n, n-1, n-$ $1, \ldots, 1,1)$ is in one-to-one correspondence with a certain set of ASM-like matrices, which also play an important role in proving the claimed identity algebraically. Finding a bijective proof remains an open problem.
\end{abstract}

Résumé. Le nombre de Triangles Monotones ayant pour dernière ligne $k_{1}<k_{2}<\cdots<k_{n}$ est donné par un polynôme $\alpha\left(n ; k_{1}, \ldots, k_{n}\right)$ en $n$ variables. Il se trouve que les valeurs de ce polynôme en les suites décroissantes $k_{1} \geq k_{2} \geq \cdots \geq k_{n}$ peuvent s'interpréter comme l'énumération signée de nouveaux objets appelés Triangles Monotones Décroissants. Il existe des liens surprenants entre ces deux classes d'objets - en particulier on prouvera l'identité $\alpha(n ; 1,2, \ldots, n)=\alpha(2 n ; n, n, n-1, n-1, \ldots, 1,1)$. En parfaite analogie avec la correspondance entre Triangles Monotones et Matrices à Signe Alternant, l'ensemble des Triangles Monotones Décroissants ayant pour dernière ligne $(n, n, n-1, n-1, \ldots, 1,1)$ est en correspondance biunivoque avec un certain ensemble de matrices similaires aux MSAs, ce qui joue un rôle important dans la preuve algébrique de l'identité précédente. C'est un problème ouvert que d'en donner une preuve bijective.

Keywords: Combinatorial Reciprocity, Monotone Triangle, Decreasing Monotone Triangle, Alternating Sign Matrix

\section{Introduction}

A Monotone Triangle of size $n$ is a triangular array of integers $\left(a_{i, j}\right)_{1 \leq j \leq i \leq n}$

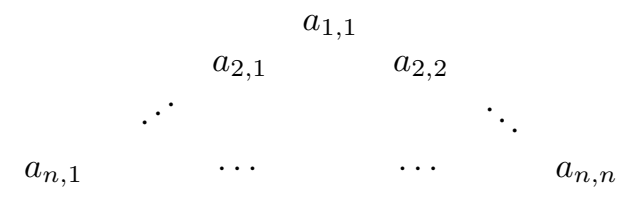

\footnotetext{
${ }^{\dagger}$ Supported by the Austrian Science Foundation FWF, START grant Y463 and NFN grant S9607-N13.

1365-8050 @ 2012 Discrete Mathematics and Theoretical Computer Science (DMTCS), Nancy, France
} 
with strict increase along rows and weak increase along North-East- and South-East-diagonals. An example of a Monotone Triangle of size 5 is given in Fig 1 . In [Fis06] it was shown that for each $n \geq 1$

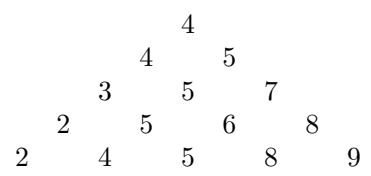

Fig. 1: One of the 16939 Monotone Triangles with bottom row $(2,4,5,8,9)$.

there exists a polynomial $\alpha\left(n ; k_{1}, k_{2}, \ldots, k_{n}\right)$ of degree $n-1$ in each of the $n$ variables, such that the evaluation of this polynomial at strictly increasing sequences $k_{1}<k_{2}<\cdots<k_{n}$ yields the number of Monotone Triangles with prescribed bottom row $\left(k_{1}, \ldots, k_{n}\right)$ - for example $\alpha(5 ; 2,4,5,8,9)=16939$. We now give an interpretation to the evaluations at weakly decreasing sequences $k_{1} \geq k_{2} \geq \cdots \geq k_{n}$ :

A Decreasing Monotone Triangle (DMT) of size $n$ is a triangular array of integers $\left(a_{i, j}\right)_{1 \leq j \leq i \leq n}$ having the following properties:

1. The entries along North-East- and South-East-diagonals are weakly decreasing.

2. Each integer appears at most twice in a row.

3. Two consecutive rows do not contain the same integer exactly once.

Note that the weak decrease along diagonals implies a weak decrease along rows. Let $\mathcal{W}_{n}\left(k_{1}, \ldots, k_{n}\right)$ denote the set of DMTs with $n$ rows and prescribed bottom row $\left(k_{1}, \ldots, k_{n}\right)$. As an example, if the bottom row is $(6,3,3,2,1)$, the right-most entry of the penultimate row has to be 2 , thus its left neighbour has to be 2 too. The second entry has to be 3 and the first entry may be 5,4 or 3 . In total $\mathcal{W}_{5}(6,3,3,2,1)$ consists of 5 DMTs (see Fig 2). A pair of adjacent identical entries in a row is briefly called pair. A pair is called duplicate-descendant (DD) if it is in the last row, or if the row below contains the same pair. The duplicate-descendants of the DMTs in Fig 2 are marked in boldface.

The first main result is Theorem 1 , which states that the evaluation of $\alpha\left(n ; k_{1}, \ldots, k_{n}\right)$ at weakly decreasing sequences $k_{1} \geq k_{2} \geq \cdots \geq k_{n}$ is a signed enumeration of DMTs with bottom row $\left(k_{1}, k_{2}, \ldots, k_{n}\right)$. A sketch of the proof can be found in Section 2

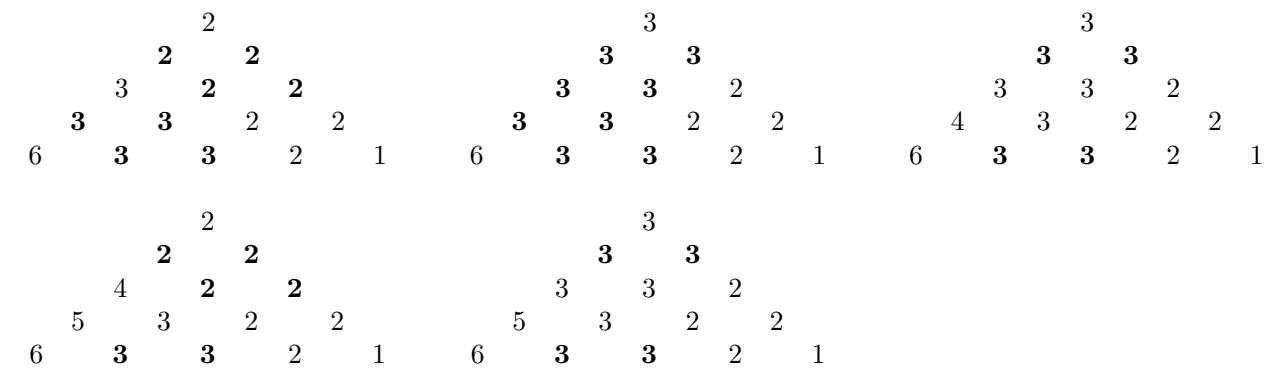

Fig. 2: The five Decreasing Monotone Triangles with bottom row $(6,3,3,2,1)$. 
Theorem 1 Let $n \geq 1$ and $k_{1} \geq k_{2} \geq \cdots \geq k_{n}$ be a sequence of weakly decreasing integers. Then

$$
\alpha\left(n ; k_{1}, \ldots, k_{n}\right)=(-1)^{\left(\begin{array}{c}
n \\
2
\end{array}\right)} \sum_{A \in \mathcal{W}_{n}\left(k_{1}, \ldots, k_{n}\right)}(-1)^{\mathrm{dd}(A)},
$$

where $\operatorname{dd}(A)$ denotes the total number of duplicate-descendants of $A$.

In our example there are four DMTs with an even number of duplicate-descendants and one with an odd number. This is consistent with Theorem 1 since $\alpha(5 ; 6,3,3,2,1)=3$.

Of special interest are Monotone Triangles with bottom row $(1,2, \ldots, n)$. This stems from the fact that they are in one-to-one correspondence with Alternating Sign Matrices (ASMs) of size n, i.e. $n \times n$ matrices with entries in $\{0,1,-1\}$ where in each row and column the non-zero entries alternate in sign and sum up to 1 . More details can be found in [Bre99]. In Section 3 we show a correspondence between DMTs with bottom row $(n, n, n-1, n-1, \ldots, 1,1)$ and a set of ASM-like matrices, which we call 2-ASMs. The reason for the interest in DMTs with this bottom row originates from computational experiments indicating that - surprisingly -

$$
\alpha(2 n ; n, n, n-1, n-1, \ldots, 1,1)=\alpha(n ; 1,2, \ldots, n)
$$

seems to hold. The second main result of the paper is in Theorem 2. In Section 4 we sketch a proof and show that Theorem 2 actually implies (2).

Theorem 2 Let $A_{n, i}$ denote the number of ASMs with the first row's unique 1 in column $i$. Then

$$
\alpha(2 n-1 ; n-1+i, n-1, n-1, \ldots, 1,1)=(-1)^{n-1} A_{n, i}
$$

holds for $i=1, \ldots, 2 n-1, n \geq 1$.

Having a combinatorial interpretation in terms of DMTs for the left-hand side of (2) and one involving Monotone Triangles for the right-hand side, the equality demands for a combinatorial explanation, i.e. a bijective proof. In Section 5 a first approach towards a bijective proof is given. The full-length version of the proofs can be found in [FR11].

\section{Decreasing Monotone Triangles}

Removing the bottom row of a Monotone Triangle with $n$ rows yields a Monotone Triangle with $n-1$ rows. If we want to count the number of Monotone Triangles with bottom row $k_{1}<k_{2}<\cdots<k_{n}$, we can thus determine all admissible penultimate rows $l_{1}<l_{2}<\cdots<l_{n-1}$ and count the number of Monotone Triangles with these bottom rows. It follows that

$$
\alpha\left(n ; k_{1}, \ldots, k_{n}\right)=\sum_{\substack{\left(l_{1}, \ldots, l_{n-1}\right) \in \mathbb{Z}^{n-1}, k_{1} \leq l_{1} \leq k_{2} \leq l_{2} \leq \cdots \leq k_{n-1} \leq l_{n-1} \leq k_{n}}} \alpha\left(n-1 ; l_{1}, \ldots, l_{n-1}\right)
$$

holds for all $k_{1}<k_{2}<\cdots<k_{n}, k_{i} \in \mathbb{Z}$. This motivates the following inductive definition of a summation operator $\sum_{\left(l_{1}, \ldots, l_{n-1}\right)}^{\left(k_{1}, \ldots, k_{n}\right)}$ for arbitrary $\left(k_{1}, \ldots, k_{n}\right) \in \mathbb{Z}^{n}$ : 


$$
\begin{aligned}
\sum_{\left(l_{1}, \ldots, l_{n-1}\right)}^{\left(k_{1}, \ldots, k_{n}\right)} A\left(l_{1}, \ldots, l_{n-1}\right):= & \sum_{\left(l_{1}, \ldots, l_{n-2}\right)}^{\left(k_{1}, \ldots, k_{n-1}\right)} \sum_{l_{n-1}=k_{n-1}+1}^{k_{n}} A\left(l_{1}, \ldots, l_{n-2}, l_{n-1}\right) \\
& +\sum_{\left(l_{1}, \ldots,,_{n-2}, l_{n-2}\right)}^{\left(k_{1}, \ldots, k_{n-1}-1\right)} A\left(l_{1}, \ldots, l_{n-2}, k_{n-1}\right), \quad n \geq 2,
\end{aligned}
$$

with $\sum_{()}^{\left(k_{1}\right)}:=$ id and the extended definition of ordinary sums

$$
\sum_{i=a}^{b} f(i):= \begin{cases}0, & b=a-1, \\ -\sum_{i=b+1}^{a-1} f(i), & b+1 \leq a-1 .\end{cases}
$$

Note that in the case of strictly increasing sequences $k_{1}<k_{2}<\cdots<k_{n}$ we have that

$$
\sum_{\left(l_{1}, \ldots, l_{n-1}\right)}^{\left(k_{1}, \ldots, k_{n}\right)} \sum_{\substack{\left(l_{1}, \ldots, l_{n-1}\right) \in \mathbb{Z}^{n-1}, k_{1} \leq l_{1} \leq k_{2} \leq l_{2} \leq \ldots \leq k_{n-1} \leq l_{n-1} \leq k_{n} \\ l_{i}<l_{i+1}}} .
$$

Use (6) and induction in (5) to see that if $A\left(l_{1}, \ldots, l_{n-1}\right)$ is a polynomial in each $l_{i}$, then there exists a polynomial $B$ in $n$ variables satisfying

$$
B\left(k_{1}, \ldots, k_{n}\right)=\sum_{\left(l_{1}, \ldots, l_{n-1}\right)}^{\left(k_{1}, \ldots, k_{n}\right)} A\left(l_{1}, \ldots, l_{n-1}\right), \quad\left(k_{1}, \ldots, k_{n}\right) \in \mathbb{Z}^{n} .
$$

Since a polynomial in $n$ variables is uniquely determined by the values on $\left\{\left(k_{1}, \ldots, k_{n}\right) \in \mathbb{Z}^{n}: k_{1}<\right.$ $\left.k_{2}<\cdots<k_{n}\right\}$, the summation operator admits the recursive description

$$
\alpha\left(n ; k_{1}, \ldots, k_{n}\right)=\sum_{\left(l_{1}, \ldots, l_{n-1}\right)}^{\left(k_{1}, \ldots, k_{n}\right)} \alpha\left(n-1 ; l_{1}, \ldots, l_{n-1}\right)
$$

for arbitrary $\left(k_{1}, \ldots, k_{n}\right) \in \mathbb{Z}^{n}$. With the same argument we now know that instead of definition (5), we can use any other inductive definition to get the same polynomial $B\left(k_{1}, \ldots, k_{n}\right)$, as long as it is based on the extended definiton of ordinary summation and coincides with the summation in (7) for all strictly increasing integer sequences $k_{1}<k_{2}<\cdots<k_{n}$. In particular, we will also use

$$
\begin{aligned}
\sum_{\left(l_{1}, \ldots, l_{n-1}\right)}^{\left(k_{1}, \ldots, k_{n}\right)} A\left(l_{1}, \ldots, l_{n-1}\right)= & \sum_{\left(l_{1}, \ldots, l_{n-2}\right)}^{\left(k_{1}, \ldots, k_{n-1}\right)} \sum_{l_{n-1}=k_{n-1}}^{\left.k_{n}, \ldots, k_{n-2}\right)} A\left(l_{1}, \ldots, l_{n-2}, l_{n-1}\right) \\
& -\sum_{\left(l_{1}, \ldots, l_{n-3}\right)}^{\left(k_{1}\right)} A\left(l_{1}, \ldots, l_{n-3}, k_{n-1}, k_{n-1}\right), \quad n \geq 3,
\end{aligned}
$$


and

$$
\begin{aligned}
\sum_{\left(l_{1}, \ldots, l_{n-1}\right)}^{\left(k_{1}, \ldots, k_{n}\right)} A\left(l_{1}, \ldots, l_{n-1}\right)= & \sum_{\left(l_{2}, \ldots, l_{n-1}\right)}^{\left(k_{2}, \ldots, k_{n}\right)} \sum_{l_{1}=k_{1}}^{k_{2}-1} A\left(l_{1}, l_{2}, \ldots, l_{n-1}\right) \\
& +\sum_{\left(l_{2}+1, k_{3}, \ldots, k_{n}\right)}^{\left(k_{n-1}\right)} A\left(k_{2}, l_{2}, \ldots, l_{n-1}\right), \quad n \geq 2 .
\end{aligned}
$$

If the bottom row $\left(k_{1}, \ldots, k_{n}\right)$ is weakly decreasing and contains three identical entries, then - by definition $-\mathcal{W}_{n}\left(k_{1}, \ldots, k_{n}\right)$ is the empty set, and thus the right-hand side of 11 is zero. Therefore we have to show that the polynomial $\alpha\left(n ; k_{1}, \ldots, k_{n}\right)$ also vanishes in this case.

Lemma 1 Let $n \geq 3$ and $A\left(l_{1}, \ldots, l_{n-1}\right)$ be a polynomial in each variable satisfying

$$
A\left(l_{1}, \ldots, l_{i-1}, l_{i}, l_{i}, l_{i}, l_{i+3}, \ldots, l_{n-1}\right)=0, \quad i=1, \ldots, n-3 .
$$

The polynomial $B\left(k_{1}, \ldots, k_{n}\right):=\sum_{\left(l_{1}, \ldots, l_{n-1}\right)}^{\left(k_{1}, \ldots, k_{n}\right)} A\left(l_{1}, \ldots, l_{n-1}\right)$ then satisfies

$$
B\left(k_{1}, \ldots, k_{i-1}, k_{i}, k_{i}, k_{i}, k_{i+3}, \ldots, k_{n}\right)=0, \quad i=1, \ldots, n-2 .
$$

Proof (sketch): Apply induction w.r.t. $n$. There are three cases to check depending on whether there are no $(i=n-2)$, one $(i=n-3)$ or at least two entries to the right of the three identical entries $(1 \leq i \leq n-4)$. All of them can be shown using (5), (9) and the induction hypothesis.

We can now restrict ourselves to the case of weakly decreasing sequences $k_{1} \geq k_{2} \geq \cdots \geq k_{n}$ which contain each integer at most twice. Note that condition 3 of DMTs is equivalent to the following condition:

$3^{\prime}$. If an entry is equal to its South-East-neighbour and smaller than its South-West-neighbour, then the entry has a right neighbour and is equal to it. If an entry is equal to its South-West-neighbour and greater than its South-East-neighbour, then the entry has a left neighbour and is equal to it.

In the following, an entry strictly smaller than the South-West-neighbour and strictly larger than the SouthEast-neighbour will be called newcomer, i.e. an entry in row $i, 1 \leq i<n$, which is not appearing in row $i+1$.

The proof of Theorem 1 consists of the following parts: In Lemma2 we show that applying the summation operator $\sum_{\left(l_{1}, \ldots, l_{n-1}\right)}^{\left(k_{1}, \ldots, k_{n}\right)}$ is a signed summation over all possible penultimate rows of DMTs with bottom row $\left(k_{1}, \ldots, k_{n}\right)$. Applying this inductively, we see in Corollary 1 that $\alpha\left(n ; k_{1}, \ldots, k_{n}\right)$ is the signed summation over all DMTs with bottom row $\left(k_{1}, \ldots, k_{n}\right)$ where the sign is determined by the total number of pairs and newcomers in the DMT without the bottom row. Finally, in Lemma 3 we show that the parity of this statistic is equal to the parity of the statistic in Theorem 1.

Lemma 2 Let $\left(k_{1}, \ldots, k_{n}\right)$ be a weakly decreasing sequence of integers with each element appearing at most twice, and let $\mathcal{P}\left(k_{1}, \ldots, k_{n}\right)$ denote the set of $(n-1)$-st rows of elements in $\mathcal{W}_{n}\left(k_{1}, \ldots, k_{n}\right)$. Then, 
for every polynomial $A\left(l_{1}, \ldots, l_{n-1}\right)$ we have

$$
\sum_{\left(l_{1}, \ldots, l_{n-1}\right)}^{\left(k_{1}, \ldots, k_{n}\right)} A\left(l_{1}, \ldots, l_{n-1}\right)=\sum_{\left(l_{1}, \ldots, l_{n-1}\right) \in \mathcal{P}\left(k_{1}, \ldots, k_{n}\right)}(-1)^{\mathrm{sc}(\mathbf{k} ; \mathbf{l})} A\left(l_{1}, \ldots, l_{n-1}\right), \quad n \geq 2,
$$

where the sign-change function $\operatorname{sc}(\mathbf{k} ; \mathbf{l}):=\operatorname{sc}\left(k_{1}, \ldots, k_{n} ; l_{1}, \ldots, l_{n-1}\right)$ is the number of pairs in $\left(l_{1}, \ldots, l_{n-1}\right)$ plus the number of newcomers.

Proof (sketch): Use induction w.r.t. $n$ and distinguish between $k_{n-1}>k_{n}$ and $k_{n-1}=k_{n}$. If $k_{n-1}>k_{n}$ use recursion (9) of the summation operator and (6). If $k_{n-1}=k_{n}$ use recursion (5) of the summation operator.

Extend the domain of the sign-changes function sc to DMTs by defining $\operatorname{sc}(A):=\sum_{i=1}^{n-1} \operatorname{sc}\left(a_{i+1,1}, \ldots, a_{i+1, i+1} ; a_{i, 1}, \ldots, a_{i, i}\right)$, where $A=\left(a_{i, j}\right)_{1 \leq j \leq i \leq n}$ is a DMT with $n$ rows.

Corollary 1 Let $k_{1} \geq k_{2} \geq \cdots \geq k_{n}$. Then

$$
\alpha\left(n ; k_{1}, \ldots, k_{n}\right)=\sum_{A \in \mathcal{W}_{n}\left(k_{1}, \ldots, k_{n}\right)}(-1)^{\mathrm{sc}(A)}
$$

holds for $n \geq 1$.

Proof: Apply $(8)$ together with Lemma 2 to inductively prove the claimed equation.

In order to complete the proof of Theorem 1 it remains to be shown that the two statistics $\operatorname{sc}(A)$ and $\left(\begin{array}{l}n \\ 2\end{array}\right)+\operatorname{dd}(A)$ have the same parity.

Lemma 3 Each $A \in \mathcal{W}_{n}\left(k_{1}, \ldots, k_{n}\right)$ satisfies $(-1)^{\operatorname{sc}(A)}=(-1)^{\left(\begin{array}{l}n \\ 2\end{array}\right)+\operatorname{dd}(A)}, \quad n \geq 1$.

Proof: By definition of DMTs, if a row contains an integer $x$ exactly once, then the row below contains $x$ either not at all - i.e. $x$ is a newcomer - or twice. In the latter case let us call $x$ a peak. Let $p(A)$ denote the number of peaks in $A, n(A)$ denote the number of newcomers in $A$ and $p_{i}(A)$ denote the number of pairs in the $i$-th row of $A$. Since newcomers and peaks are by definition not in the bottom row and every entry of a DMT not in the bottom row is either a peak, a newcomer or in a pair, it follows that

$$
\left(\begin{array}{l}
n \\
2
\end{array}\right)=p(A)+n(A)+2 \sum_{i=1}^{n-1} p_{i}(A) \equiv p(A)+n(A) \quad \bmod 2 .
$$

Let us call a pair $(x, x)$ a base-pair if it is located in the bottom row, or the row below contains $x$ exactly once. Let $b(A)$ denote the number of base-pairs in $A$. By definition, duplicate-descendants are those pairs, which are either in the bottom row or not a base-pair. Further note that the set of peaks and the set of base-pairs are in one-to-one correspondence (see Fig 3). Hence, we have

$$
\operatorname{dd}(A)=\sum_{i=1}^{n} p_{i}(A)-b(A)+p_{n}(A) \equiv \sum_{i=1}^{n-1} p_{i}(A)-p(A) \quad \bmod 2 .
$$

Since $\operatorname{sc}(A)=n(A)+\sum_{i=1}^{n-1} p_{i}(A)$ the claimed equality follows. 


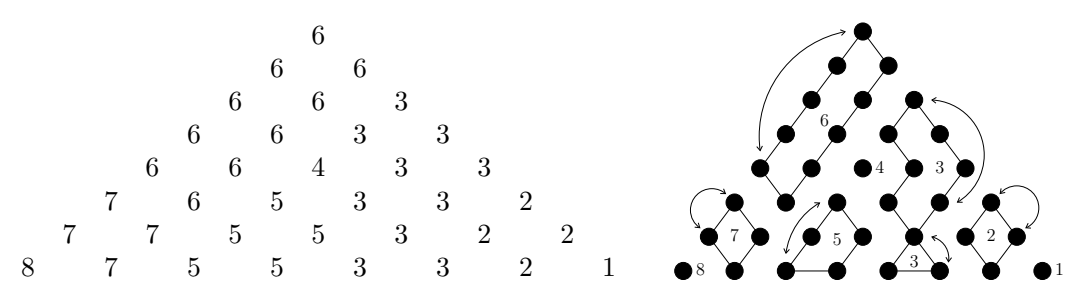

Fig. 3: A DMT and its structural decomposition.

\section{DMTs and 2-ASMs}

In order to describe the impact of the change from Monotone Triangles to DMTs on the level of matrices, one may consider the following equivalent definition of ASMs: An Alternating Sign Matrix (ASM) of size $n$ is an $n \times n$-matrix where each row and each column is a word of the ASM-machine in Fig 4(a). From this vantage point we give the following definition of 2-ASMs: A 2-ASM of size $n$ is a $(2 n) \times n$ matrix where each row is a word of the ASM-machine in Fig 4(a) and each column is a word of the 2-ASM-machine in Fig 4(b), In words, the non-zero entries of columns occur in pairs, such that each partial column sum is in $\{0,1,2\}$, and each column sums up to 2 .

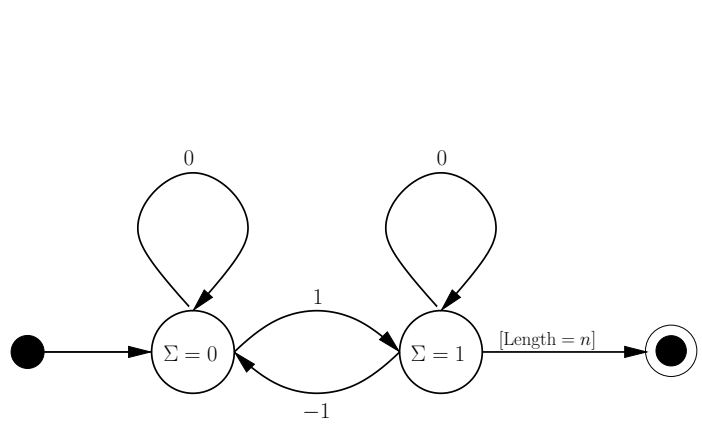

(a)

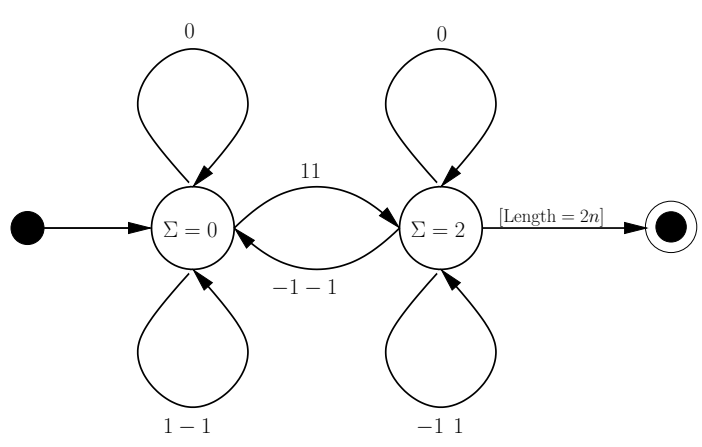

(b)

Fig. 4: The ASM-machine generating the rows and columns of ordinary ASMs of size $n$ on the left-hand side and the 2-ASM-machine generating the columns of 2-ASMs of size $n$ on the right-hand side.

Theorem 3 The set $\mathcal{W}_{2 n}(n, n, n-1, n-1, \ldots, 1,1)$ is in one-to-one correspondence with the set of 2-ASMs of size $n$.

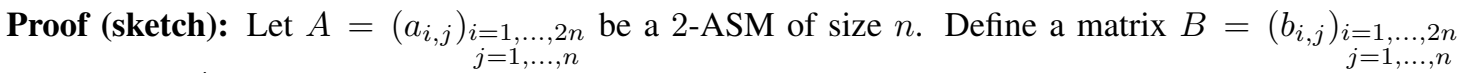
by $b_{i, j}=\sum_{k=1}^{i} a_{k, j}$. Each of the first $i$ rows of $A$ sum up to 1 , thus the $i$-th row of $B$ sums up to $i$. By definition of the 2-ASM-machine, the first $i$ entries of each column sum up to 0,1 or 2 , thus $b_{i, j} \in\{0,1,2\}$. Hence, one can define a triangular array $T$ of integers in $\{1,2, \ldots, n\}$ with $2 n$ rows where each row is weakly decreasing, by specifying that row $i$ contains $b_{i, j}$ entries $j$. It is not difficult to check that $T \in \mathcal{W}_{2 n}(n, n, n-1, n-1, \ldots, 1,1)$ and that this mapping is indeed a bijection. 


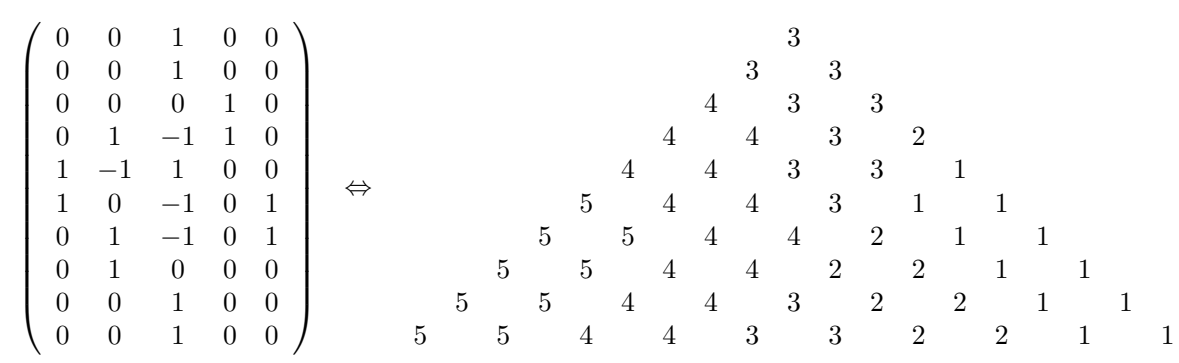

Fig. 5: A 2-ASM of size 5 and the corresponding DMT.

An example of corresponding objects can be seen in Fig 5

\section{Connections between Monotone Triangles and DMTs}

The starting point for the content of this section was the empirical observation of [2]. The algebraic proof in this section uses a methodology which was successfully applied in [Fis07], where the Refined ASM Theorem is reproven: The number $A_{n, i}$ of ASMs of size $n$ with the first column's unique 1 situated in row $i$ is equal to $\left(\begin{array}{c}n+i-2 \\ n-1\end{array}\right) \frac{(2 n-i-1) !}{(n-i) !} \prod_{k=0}^{n-2} \frac{(3 k+1) !}{(n+k) !}$. The methodology consists of the following steps:

- Denoting $E_{x} f(x):=f(x+1)$ and $\Delta_{x} f(x):=f(x+1)-f(x)=\left(E_{x}-\mathrm{id}\right) f(x)$, show that

$$
A_{n, i}=\left.(-1)^{i-1} \Delta_{k_{1}}^{i-1} \alpha\left(n ; k_{1}, 2,3, \ldots, n\right)\right|_{k_{1}=2} .
$$

- Derive a system of linear equations for $A_{n, i}$ implying that $\left(A_{n, i}\right)_{i=1, \ldots, n}$ is an eigenvector of a certain matrix.

- Show that the corresponding eigenspace is one-dimensional.

- Show that the conjectured numbers are in this eigenspace too.

- Determine the constant factor by which they differ to be 1.

We can apply this methodology to give a proof of Theorem 4

Theorem 4 The numbers $W_{n, i}:=\left.\Delta_{k_{1}}^{i-1} \alpha\left(2 n-1 ; k_{1}, n-1, n-1, n-2, n-2, \ldots, 1,1\right)\right|_{k_{1}=n}$ are given by

$$
W_{n, i}=\sum_{l=1}^{i}\left(\begin{array}{l}
i-1 \\
l-1
\end{array}\right)(-1)^{n+i+l-1} A_{n, l}
$$

for all $i=1, \ldots, 2 n-1, n \geq 1$.

It is not difficult to see that Theorem 4 and Theorem 2 are equivalent. Applying Lemma 2 to $\alpha(2 n+$ $1 ; n+1, n, n, \ldots, 1,1)$ one can also see that Equation 2) is a consequence of Theorem 2 .

The proof of Theorem 4 consists of the following parts: In Lemma 4 we show that the numbers $W_{n, i}$ satisfy a certain symmetry relation by giving them a combinatorial meaning. In Lemma 5$]$ we derive that $\left(W_{n, i}\right)_{i=1, \ldots, 2 n-1}$ is an eigenvector of $\left(\left(\begin{array}{c}n-i \\ 2 n-i-j\end{array}\right)(-1)^{n+i}\right)_{1 \leq i, j \leq 2 n-1}$. In Lemma 6 it is shown that the 
corresponding eigenspace is one-dimensional. In Lemma 7 we deduce that $\left(\sum_{l=1}^{i}\left(\begin{array}{c}i-1 \\ l-1\end{array}\right)(-1)^{n+i+l-1} A_{n, l}\right)_{i=1, \ldots, 2 n-1}$ is in the same eigenspace. In Lemma 8 we show a recursion for $W_{n, 1}$, which lets us then inductively derive the constant factor.

Lemma 4 The numbers $W_{n, i}$ satisfy the symmetry relation

$$
W_{n, i}=(-1)^{n-1} W_{n, 2 n-i}
$$

for $i=1, \ldots, 2 n-1, n \geq 1$.

Proof (sketch): Using recursion (10, , (8) and Lemma 2 one can show for weakly decreasing sequences $k_{1} \geq k_{2} \geq \cdots \geq k_{n}$ that

$$
\Delta_{k_{1}} \alpha\left(n ; k_{1}, \ldots, k_{n}\right)=-\sum_{\left(l_{2}, \ldots, l_{n-1}\right) \in \mathcal{P}\left(k_{2}, \ldots, k_{n}\right)}(-1)^{\mathrm{sc}\left(k_{2}, \ldots, k_{n} ; l_{2}, \ldots, l_{n-1}\right)} \alpha\left(n-1 ; k_{1}, l_{2}, \ldots, l_{n-1}\right),
$$

where $\operatorname{sc}\left(k_{2}, \ldots, k_{n} ; l_{2}, \ldots, l_{n-1}\right)$ is the total number of pairs and newcomers in $\left(l_{2}, \ldots, l_{n-1}\right)$.

A DMT-trapezoid with bottom row $\left(k_{1}, \ldots, k_{n}\right)$ and top row $\left(l_{1}, \ldots, l_{n-i+1}\right)$ is an array of integers $\left(a_{p, q}\right)_{\substack{1 \leq p \leq i, 1 \leq q \leq n-i+p}}$ having the same properties as DMTs. Let $\mathcal{P}_{i}(\mathbf{k} ; \mathbf{l})$ denote the set of DMT-trapezoids with $i$ rows, bottom row $\mathbf{k}$ and top row $\mathbf{l}$. As the $\Delta$-operator is linear and the sequence $\left(k_{1}, l_{2}, \ldots, l_{n-1}\right)$ is again weakly decreasing, we can apply induction and Corollary 1 to see that

$$
W_{n, i}=\sum_{\substack{\left(l_{1}, \ldots, l_{2 n-i-1}, \bigcap_{i}\right): \\ \mathcal{P}_{i}\left(n-1, n-1, \ldots, 1,1 ; l_{1}, \ldots, l_{2 n-i-1}\right)}} \sum_{\Delta \in \mathcal{W}_{2 n-i}\left(n, l_{1}, l_{2}, \ldots, l_{2 n-i-1}\right)}(-1)^{i-1+\operatorname{sc}(\triangle)+\operatorname{sc}(\triangle)},
$$

where $\operatorname{sc}(\square)$ denotes the total number of pairs and newcomers without the bottom row in the DMTtrapezoid. Hence, $W_{n, i}$ is a signed enumeration of DMT-trapezoids with $i$ rows, bottom row $(n-1, n-$ $1, \ldots, 1,1)$ where in the top row an entry $n$ is added at the left end and completed to the top as DMT. From Theorem 3 it follows that these objects are in one-to-one correspondence with the following $(2 n-$ 1) $\times(n-1)$-matrices: all columns are generated by the machine in Fig 4(b) (with length $2 n-1)$, and all rows - with the exception of the $(2 n-i)$-th row - are generated by the machine in Fig 4(a) (with length $n-1)$. The $(2 n-i)$-th row is generated by the machine in Fig 4(a) (with length $n-1)$ with the modification that the transition to the end state is at the state $\Sigma=0$. An example can be seen in Fig 6 . Reflecting the matrices along the horizontal symmetry axis yields the one-to-one correspondence between

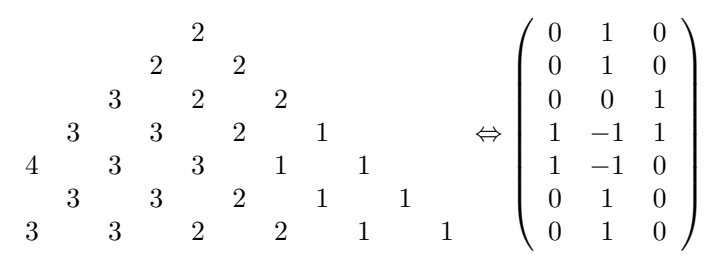

Fig. 6: One of the objects enumerated by $W_{4,3}$ and its corresponding matrix.

the objects enumerated by $W_{n, i}$ and those enumerated by $W_{n, 2 n-i}$. Using the interpretation of pairs and 
newcomers in terms of edges taken in the 2-ASM machine in Fig 4(b) one can show that the parity of $\operatorname{sc}(\triangle)+\operatorname{sc}(\triangle)$ changes by $n-1$ under this reflection.

Lemma 5 Fix $n \geq 1$. The numbers $W_{n, i}$ satisfy the system of linear equations

$$
W_{n, i}=\sum_{k=1}^{2 n-1}\left(\begin{array}{c}
n-i \\
2 n-i-k
\end{array}\right)(-1)^{n+i} W_{n, k}
$$

for $i=1, \ldots, 2 n-1$.

Proof (sketch): We will make use of the following properties of the $\alpha$-polynomial

1. $\alpha\left(n ; k_{1}, k_{2}, \ldots, k_{n}\right)=(-1)^{n-1} \alpha\left(n ; k_{2}, k_{3}, \ldots, k_{n}, k_{1}-n\right)$

2. $\alpha\left(n ; k_{1}, k_{2}, \ldots, k_{n}\right)=\alpha\left(n ;-k_{n},-k_{n-1}, \ldots,-k_{1}\right)$

3. $\alpha\left(n ; k_{1}, k_{2}, \ldots, k_{n}\right)=\alpha\left(n ; k_{1}+c, k_{2}+c, \ldots, k_{n}+c\right), \quad c \in \mathbb{Z}$.

The first property was shown in [Fis07], Lemma 5. The second and third property is clear for strictly increasing sequences $k_{1}<k_{2}<\cdots<k_{n}$. The polynomiality implies the equality for arbitrary $\left(k_{1}, \ldots, k_{n}\right) \in \mathbb{Z}^{n}$. It follows that

$$
W_{n, i}=\left.E_{k_{1}}^{i-n} \delta_{k_{1}}^{i-1} \alpha\left(2 n-1 ; n-1, n-1, n-2, n-2, \ldots, 1,1, k_{1}-n\right)\right|_{k_{1}=n},
$$

where $\delta_{x} f(x):=f(x)-f(x-1)=\left(\mathrm{id}-E_{x}^{-1}\right) f(x)=E_{x}^{-1} \Delta_{x} f(x)$. Using $\delta_{x}^{i} f(-x)=\left.(-1)^{i} \Delta_{y}^{i} f(y)\right|_{y=-x}$ and the symmetry shown in Lemma 4 one can deduce the claimed equation.

Lemma 6 Let $\delta_{i, j}$ denote the Kronecker delta. Then

$$
\operatorname{rk}\left(\left(\begin{array}{c}
n-i \\
2 n-i-j
\end{array}\right)(-1)^{n+i}-\delta_{i, j}\right)_{1 \leq i, j \leq 2 n-1}=2 n-2
$$

holds for $n \geq 1$.

Proof (sketch): Removing the $n$-th row and the last column results in a $(2 n-2) \times(2 n-2)$-matrix, which turns out to have determinant equal to $(-1)^{n-1} \operatorname{det}_{0 \leq i, j \leq n-2}\left(\left(\begin{array}{c}i+j \\ j\end{array}\right)-\delta_{i, j+1}\right)$. In [BFZJ11] it was shown that the determinant of $\left(\left(\begin{array}{c}i+j \\ j\end{array}\right)-\delta_{i, j+1}\right)_{0 \leq i, j \leq n-1}$ equals the number of descending plane partitions with each part smaller than or equal to $n$, which is known to be equal to the number of ASMs of size $n$.

Lemma 7 Let $X_{n, i}:=\sum_{j=1}^{i}\left(\begin{array}{c}i-1 \\ j-1\end{array}\right)(-1)^{n+i+j-1} A_{n, j}$. Then

$$
\sum_{k=1}^{2 n-1}\left(\begin{array}{c}
n-i \\
2 n-i-k
\end{array}\right)(-1)^{n+i} X_{n, k}=X_{n, i}
$$

holds for all $i=1, \ldots, 2 n-1, n \geq 1$. 
Proof (sketch): Using $\left(\begin{array}{l}n \\ k\end{array}\right)=\left(\begin{array}{c}n \\ n-k\end{array}\right),\left(\begin{array}{l}a \\ b\end{array}\right)=\left(\begin{array}{c}b-a-1 \\ b\end{array}\right)(-1)^{b}$ and Chu-Vandermonde convolution, one can see that it suffices to show that

$$
\begin{gathered}
\left(\sum_{j=1}^{2 n-1}\left(\begin{array}{c}
n+j-2 \\
n-1
\end{array}\right)\left(\begin{array}{c}
2 n-j-1 \\
n-1
\end{array}\right)\left(\begin{array}{c}
n-i-j \\
2 n-i-j
\end{array}\right)\right)_{i=1, \ldots, 2 n-1} \\
=\left(\sum_{j=1}^{2 n-1}\left(\begin{array}{c}
i-1 \\
j-1
\end{array}\right)\left(\begin{array}{c}
n+j-2 \\
n-1
\end{array}\right)\left(\begin{array}{c}
2 n-j-1 \\
n-1
\end{array}\right)(-1)^{n+j}\right)_{i=1, \ldots, 2 n-1}
\end{gathered}
$$

This can be done by multiplying with the invertible matrix $T:=\left(\left(\begin{array}{c}i-1 \\ j-1\end{array}\right)(-1)^{j}\right)_{1<i, j<2 n-1}$ from the left on both sides and using the identity $\left(\begin{array}{c}n+j-2 \\ n-1\end{array}\right)\left(\begin{array}{c}2 n-j-1 \\ n-1\end{array}\right)\left(\begin{array}{c}n+i-2 \\ 2 n-j-1\end{array}\right)=\left(\begin{array}{c}n+j-2 \\ j-1\end{array}\right)\left(\begin{array}{c}i-1 \\ n-j\end{array}\right)\left(\begin{array}{c}n+i-2 \\ n-1\end{array}\right)$.

Lemma 8 The numbers $W_{n, i}$ satisfy the equation

$$
W_{n, 1}=-\sum_{i=1}^{n-1}\left(\begin{array}{c}
n-1 \\
i
\end{array}\right) W_{n-1, i}
$$

for all $n \geq 2$.

Proof (sketch): Using Lemma 2 and $\alpha\left(n ; k_{1}, \ldots, k_{n}\right)=(-1)^{n-1} \alpha\left(n ; k_{n}+n, k_{1}, \ldots, k_{n-1}\right)$ (compare to the first property of $\alpha$ in the proof of Lemma 5), we have

$$
W_{n, 1}=(-1)^{n} \alpha(2 n-2 ; 2 n-1, n-1, n-1, \ldots, 2,2,1) .
$$

Apply Lemma 2 together with the binomial identity $\sum_{k=n}^{m}\left(\begin{array}{l}k \\ n\end{array}\right)=\left(\begin{array}{l}m+1 \\ n+1\end{array}\right)$ to see the claimed equation.

Using induction w.r.t. $n$ we can conclude the proof of Theorem 4 by showing that $X_{n, i}=W_{n, i}$ for all $i=1, \ldots, 2 n-1$. Since both $\left(X_{n, i}\right)_{i=1, \ldots, 2 n-1}$ and $\left(W_{n, i}\right)_{i=1, \ldots, 2 n-1}$ are in the same one-dimensional eigenspace for fixed $n \geq 1$, it follows that $\left(X_{n, i}\right)_{i=1, \ldots, 2 n-1}=C_{n}\left(W_{n, i}\right)_{i=1, \ldots, 2 n-1}$. Hence, it suffices to show that $X_{n, 1}=W_{n, 1}$. This can be done by applying Lemma 8 , the induction hypothesis and ChuVandermonde convolution.

\section{Towards a bijective proof}

It follows by Theorem 1 that proving equation $[2]$ is equivalent to showing that

$$
\sum_{A \in \mathcal{W}_{2 n}(n, n, n-1, n-1, \ldots, 1,1)}(-1)^{\overline{d d}(A)}=\alpha(n ; 1,2, \ldots, n), \quad n \geq 1,
$$

where $\overline{d d}(A)$ is the number of pairs $(x, x)$, for which there also exists a pair $(x, x)$ in the row below.

A bijective proof of 15 ) could succeed by partitioning $\mathcal{W}_{2 n}(n, n, n-1, n-1, \ldots, 1,1)$ into three sets $S_{1}, S_{2}$ and $S_{3}$, such that all elements of $S_{1}$ as well as $S_{2}$ have even $\overline{d d}$-parity, whereas all elements of $S_{3}$ 
have odd $\overline{d d}$-parity. The elements of $S_{1}$ should be in bijective correspondence with Monotone Triangles with bottom row $(1,2, \ldots, n)$, and the elements of $S_{2}$ and $S_{3}$ should be in bijective correspondence.

It is plausible that the subset $S_{1} \subseteq \mathcal{W}_{2 n}(n, n, n-1, n-1, \ldots, 1,1)$ corresponding to the Monotone Triangles, is given by those DMT with bottom row $(n, n, n-1, n-1, \ldots, 1,1)$ where the $(2 i)$-th row consists of $i$ pairs for all $i=1, \ldots, n$ (see Fig 7(a) . Note that this also determines the entries in odd rows. Identifying the entries connected by an edge with one single entry and reflecting the triangle along the vertical symmetry axis yields the one-to-one correspondence with Monotone Triangles. Since for each $A \in S_{1}$ we have that $\overline{d d}(A)$ is twice the number of diagonally adjacent identical entries in the corresponding Monotone Triangle, it follows that $(-1)^{\overline{d d}(A)}=1$. Finding a sign-reversing involution on $\mathcal{W}_{2 n}(n, n, n-1, n-1, \ldots, 1,1) \backslash S_{1}$ remains an open problem.

From the viewpoint of 2-ASMs the subset $S_{1} \subseteq \mathcal{W}_{2 n}(n, n, n-1, n-1, \ldots, 1,1)$ corresponds to the set of 2-ASMs where the column generation is restricted to the machine in Fig7(b) The one-toone correspondence with Monotone Triangles is even more obvious from this vantage point. Using this connection might turn out to be useful in finding a bijection.

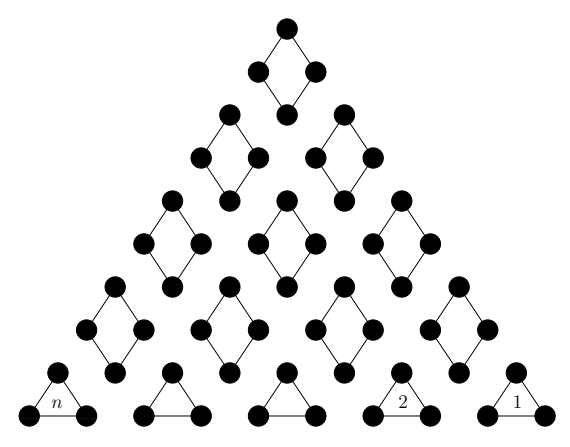

(a)

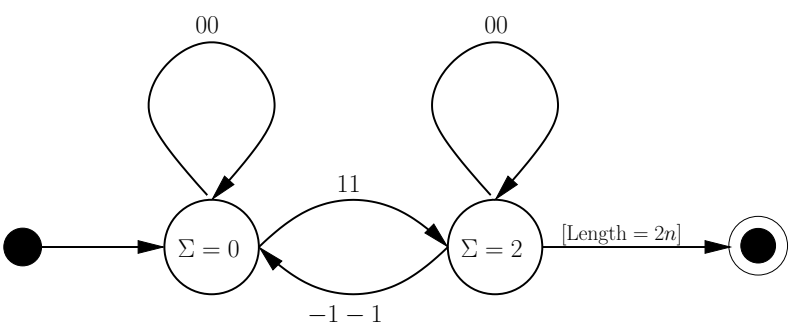

(b)

Fig. 7: Structural restriction of DMTs such that they correspond to Monotone Triangles.

\section{References}

[BFZJ11] R. E. Behrend, P. Di Francesco, and P. Zinn-Justin. On the weighted enumeration of alternating sign matrices and descending plane partitions. arXiv:1103.1176v1, 2011.

[Bre99] D. M. Bressoud. Proofs and Confirmations: The Story of the Alternating-Sign Matrix Conjecture. Cambridge University Press, 1999.

[Fis06] I. Fischer. The number of monotone triangles with prescribed bottom row. Adv. Appl. Math., no.2, 37:249-267, 2006.

[Fis07] I. Fischer. A new proof of the refined alternating sign matrix theorem. J. Comb. Theory Ser. A, 114:253-264, 2007.

[FR11] I. Fischer and L. Riegler. Combinatorial reciprocity for monotone triangles. arXiv:1111.2695v1, 2011. 